\title{
Constructions for 4-Polytopes and the Cone of Flag Vectors
}

\author{
Andreas Paffenholz and Axel Werner
}

\begin{abstract}
We describe a construction for $d$-polytopes generalising the well known stacking operation. The construction is applied to produce 2-simplicial and 2-simple 4-polytopes with $g_{2}=0$ on any number of $n \geq 13$ vertices. In particular, this implies that the ray $\ell_{1}$, described by Bayer (1987), is fully contained in the convex hull of all flag vectors of 4-polytopes. Especially interesting examples on 9,10 and 11 vertices are presented.
\end{abstract}

\section{Introduction}

It is a wide open problem in discrete geometry to understand the combinatorial properties of polytopes, which can be described as the convex hull of finitely many points in some $\mathbb{R}^{n}$. Even the more special task to characterise the $f$ - and flag vectors of polytopes (and more generally of spheres) of arbitrary dimension seems to be very hard. For 3-dimensional polytopes, Steinitz Ste06 gave a complete characterisation. For arbitrary dimension, the problem is still open, although some conditions are known. In particular, all linear relations between the entries of flag vectors are described by the Generalized Dehn-Sommerville equations BB85 and the admissible $f$-vectors of simplicial polytopes are classified in terms of their $g$ vectors by the $g$-theorem of Billera, Lee and Stanley (see Zie95 Thm. 8.35]). Additionally, for dimension 4 a linear approximation of the set of flag vectors and $f$-vectors was given by Bayer Bay87. A different view on the $f$-vector characterisation was provided by Ziegler Zie02. Since then, some progress has been made (cf. PZ04, Zie04), but it is still not known what the linear cone of $f$-resp. flag vectors looks like.

In this paper we introduce a new polytope construction method, examine some of its combinatorial properties and construct 4-polytopes with arbitrarily high numbers of vertices that are extremal for the flag vector cone. In particular, we prove the following two theorems (see below for definitions).

2000 Mathematics Subject Classification. Primary 52B05;52B12.

Key words and phrases. Polytope, Flag Vector, Flag Vector Cone, $g$-Vector, Elementary Polytope, 2-Simple, 2-Simplicial.

The first author was supported by the Deutsche Forschungsgemeinschaft within the European graduate program 'Combinatorics, Geometry, and Computation' (No. GRK 588/2).

The second author was supported by Mr Leibniz. 
Theorem 4.3, Elementary 2-simplicial, 2-simple 4-polytopes with $k$ vertices exist for $k=5,9,10,11$ and $k \geq 13$.

This implies (using the notation of Bayer Bay87):

Corollary 4.4. The ray $\ell_{1}$ is contained in the convex hull of all flag vectors of 4-polytopes.

Additionally, we briefly analyse the consequences of the various recent polytope constructions in PZ04 and Zie04 for the flag vector cone.

1.1. General preliminaries. We first give the basic definitions as well as an overview over important related results.

Let $P$ be a $d$-polytope and $[d]=\{0, \ldots, d-1\}$. The $f$-vector of $P$ is the $d$ dimensional vector

$$
f(P)=\left(f_{0}, f_{1}, \ldots, f_{d-1}\right),
$$

where $f_{i}$ for $0 \leq i \leq d-1$ denotes the number of $i$-dimensional faces of $P$. The flag vector of $P$ is the $2^{d}$-dimensional vector

$$
\operatorname{flag}(P)=\left(f_{S}\right)_{S \subseteq[d]},
$$

where $f_{S}$ for a subset $S=\left\{i_{1}, \ldots, i_{k}\right\}$ of $[d]$ denotes the number of face chains $\emptyset \subset F_{i_{1}} \subset \ldots \subset F_{i_{k}} \subset P$ such that $\operatorname{dim} F_{i_{j}}=i_{j}$ for $1 \leq j \leq k$. We usually write $f_{i_{1} i_{2} \ldots i_{k}}$ instead of $f_{\left\{i_{1}, i_{2}, \ldots, i_{k}\right\}}$. Faces of codimension 1, 2 and 3 are called facets, ridges and subridges respectively.

The Generalized Dehn-Sommerville equations by Bayer and Billera BB85 imply that the flag vectors of $d$-polytopes lie in an $\left(F_{d}-1\right)$-dimensional affine subspace of $\mathbb{R}^{2^{d}}$, where $\left(F_{k}\right)_{k \geq 0}=(1,1,2,3, \ldots)$ is the series of Fibonacci numbers.

$P$ is $k$-simplicial $(0 \leq k \leq d-1)$ if all its $k$-dimensional faces are $k$-simplices, i.e. contain exactly $k+1$ vertices; equivalently, if in the face lattice of $P$ all intervals $[\emptyset, F]$ for $\operatorname{dim} F=k$ are boolean.

$P$ is $h$-simple $(0 \leq h \leq d-1)$ if the dual polytope $P^{\Delta}$ is $h$-simplicial; equivalently, if all intervals $[F, P]$ with $\operatorname{dim} F=d-h-1$ are boolean or equivalently, if all its $(d-h-1)$-dimensional faces are contained in exactly $h+1$ facets.

The $g$-vector of a polytope can be defined in general using generating functions (see Sta97); its entries can be written as linear combinations of the entries of the flag vector. We will, however, focus on one special entry.

Definition 1.1. For a $d$-polytope $P$ define

$$
g_{2}(P)=f_{02}-3 f_{2}+f_{1}-d f_{0}+\left(\begin{array}{c}
d+1 \\
2
\end{array}\right) .
$$

It has been shown by Kalai $\mathbf{K a l 8 7}$ via rigidity theory that $g_{2}(P) \geq 0$ for every $d$-polytope $P$ with $d \geq 4$. Polytopes $P$ with $g_{2}(P)=0$ are called elementary. It is an interesting open problem to characterise all elementary polytopes. See also Kal94 for a survey on this topic.

1.2. Flag vectors of 4-polytopes. In the last two sections we will focus on 4-polytopes, where the situation is understood slightly better than in general. It is easy to prove that for 2-simplicial, 2 -simple 4-polytopes the $f$-vector is symmetric and the flag vector is completely determined by the $f$-vector.

Bayer Bay87 described all the known linear inequalities for flag vectors of 4polytopes. Let $\mathcal{D} \subseteq \mathbb{R}^{4}$ be the polyhedron defined by these inequalities and $\mathcal{C} \subseteq \mathcal{D}$ 


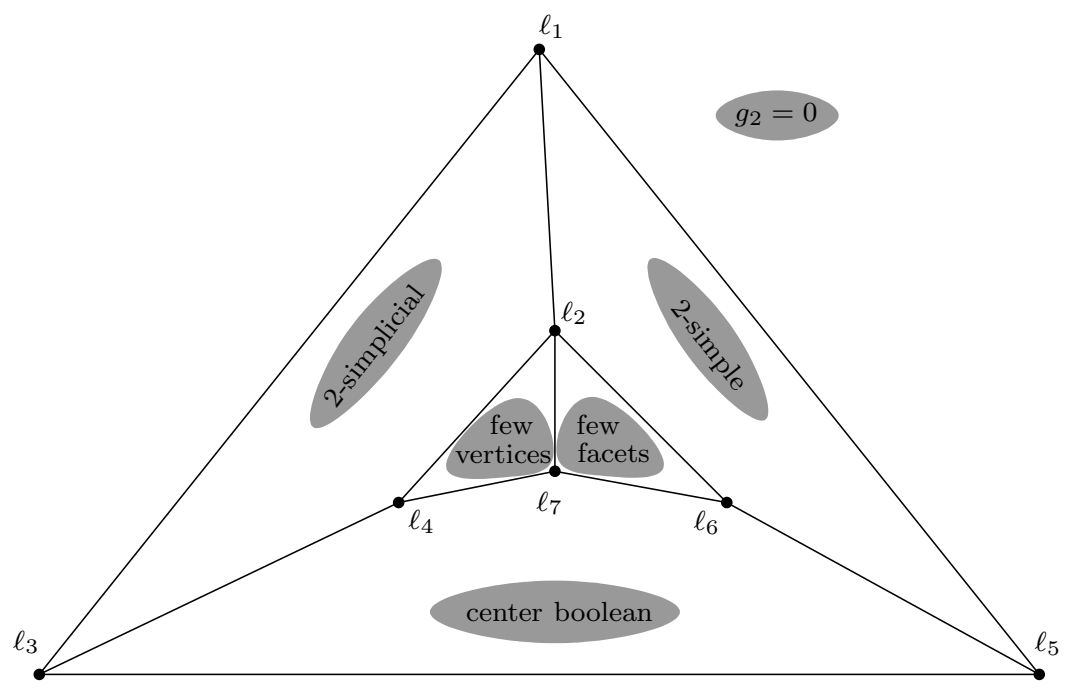

FiguRE 1.1. Hyperplane section through the cone $\mathcal{D}$, according to Bay87

the convex hull of all flag vectors of 4-polytopes. Then $\mathcal{D}$ is a 4 -dimensional cone with the flag vector of the 4-simplex as its apex. Figure 1.1 illustrates a hyperplane section through this cone. It can be viewed as a 3-polytope with its vertices, edges and faces representing special properties of 4-polytopes, in the sense that polytopes whose flag vectors lie on the respective faces have these properties. The main question is how close the cone $\mathcal{D}$ approximates $\mathcal{C}$. The task is therefore to find examples of polytopes with flag vectors in extremal regions of $\mathcal{D}$.

In Table 1.1 we give a summary of what is known for the rays of $\mathcal{D}$; note that $\ell_{4}$ and $\ell_{6}$ are not contained in $\mathcal{C}$ itself, but in its closure (cf. Bay87, Sec. 2]).

Until now only two flag vectors of polytopes on $\ell_{1}$ were known, that of the 4 -simplex $\Delta_{4}$ and that of the 4-dimensional hypersimplex $\Delta_{4}(2)$ (for a definition see Zie95. Ch. 0] for instance). Hence it was clear that $\ell_{1}$ contained an edge of $\mathcal{C}$, but not, whether $\ell_{1}$ is a ray of $\mathcal{C}$, nor if $\ell_{1}$ contained any further flag vectors at all. We establish in Section 4 that $\ell_{1}$ is indeed an extremal ray of $\mathcal{C}$.

A few more regions of $\mathcal{D}$ deserve a closer study. The set of flag vectors of general 2simplicial, 2-simple 4-polytopes is a subset of the 2-dimensional cone spanned by $\ell_{1}$ and $\ell_{2}$. It is indeed a 2 -dimensional set - there are 2 -simplicial, 2 -simple 4 -polytopes

TABLE 1.1. Known polytopes on or close to the rays of $\mathcal{D}$

\begin{tabular}{cll} 
ray & property & examples \\
\hline$\ell_{1}$ & 2 -simplicial, 2-simple 4-polytopes with $g_{2}=0$ & see Theorem 4.3 \\
$\ell_{2}$ & 'fat' 2-simplicial, 2-simple 4-polytopes & unknown \\
$\ell_{7}$ & 'fat' center boolean 4-polytopes & unknown \\
$\ell_{4}$ & simplicial 4-polytopes with few vertices & cyclic polytopes \\
$\ell_{3}$ & simplicial 4-polytopes with $g_{2}=0$ & stacked polytopes \\
$\ell_{5}$ & simple 4-polytopes with $g_{2}=0$ & truncated polytopes \\
$\ell_{6}$ & simple 4-polytopes with few facets & dual cyclic polytopes
\end{tabular}


with the same number of vertices, but different numbers of edges (cf. [PZ04). Analogously, the set of flag vectors of elementary 4-polytopes is contained in the 3 -dimensional cone spanned by $\ell_{1}, \ell_{3}$ and $\ell_{5}$. There are also a number of extreme examples: stacked polytopes, iterated pyramids over $n$-gons and multiplexes (cf. BBS02]). Also, the constructions described in the next sections produce in general elementary polytopes when applied to such, as can be seen from Corollary 3.8 Note that 2-simplicity, 2-simpliciality and $g_{2}=0$ are three independent properties, that is, there are 4-polytopes with any combination of these properties. Bayer conjectured that the hyperplane determined by $\ell_{2}, \ell_{4}$ and $\ell_{6}$ yields a valid inequality for 4-polytopes. This is not true, since there exist polytopes with flag vectors close to the interior of the edges $\left[\ell_{4}, \ell_{7}\right]$, and dually $\left[\ell_{6}, \ell_{7}\right]$, as shown by Joswig \& Ziegler JZZ00 and Ziegler Zie04. However, a hyperplane cutting off $\ell_{7}$ may still be possible. The most extremal polytopes known in this respect are projected products of polygons by Ziegler Zie04 and polytopes obtained by the $E$ construction (Paffenholz \& Ziegler [PZ04]). The most restrictive linear inequality that is compatible with these examples would then be

$$
f_{03}-140 \geq 4\left(f_{1}+f_{2}\right)-20\left(f_{0}+f_{3}\right) .
$$

In terms of the fatness $\mathrm{F}$ and complexity $\mathrm{C}$ of 4-polytopes, as introduced by Ziegler Zie02, this inequality reads $4 \mathrm{~F}-\mathrm{C} \leq 20$. In this respect, providing an upper bound for fatness, that is, bounding the number of edges and ridges by the number of vertices and facets, would be helpful.

\section{General Construction}

This section consists of two parts. In the first part we provide the basic tool for the construction of a family of 2 -simple and 2-simplicial 4-polytopes with vanishing $g_{2}$. The operation has many more applications than the ones we will discuss in more detail in the second part. Hence, we give all definitions and theorems for arbitrary dimension $d \geq 3$. We indicate some of the additional applications we have encountered so far.

In the second part we examine settings in which the operation can be applied, determine the facet types that can occur by the construction and prove that some interesting properties of polytopes are preserved. This part will be much more specifically tailored for what we need for the polytope families defined later, as some of the $f$-vector computations tend to get complicated in a more general setting. In Section 3 we combine several instances of our basic tool to obtain two special constructions $\mathcal{I}^{1}$ and $\mathcal{I}^{2}$ producing 4-polytopes $\mathcal{I}^{1}(P ; S)$ and $\mathcal{I}^{2}(P ; S)$ out of a polytope $P$ and a facet $S$ of $P$. Using the properties of the construction discussed in this section we prove that 2-simplicity, 2-simpliciality and the value of $g_{2}$ is preserved by the construction.

2.1. Pseudo-Stacking. The operation we introduce here is a generalisation of the well known stacking operation. In most cases it adds one new vertex to the polytope. We need a couple of new notations for this.

Definition 2.1. Let $P$ be a $d$-polytope. A simplex facet of $P$ is a facet of $P$ that is combinatorially equivalent to a $(d-1)$-simplex. 
Let $H:=\left\{x \in \mathbb{R}^{d} \mid\langle x, v\rangle=\ell\right\}$ for some $v \in \mathbb{R}^{d}$ and $\ell \in \mathbb{R}$ be an affine hyperplane. By $H^{+}:=\left\{x \in \mathbb{R}^{d} \mid\langle x, v\rangle>\ell\right\}$ we denote the positive half space defined by $H$. Similarly, we denote the negative half space by $H^{-}$.

Let $F$ be a facet of a $d$-polytope $P$. We denote by $H_{F}$ the unique affine hyperplane that contains $F$, oriented in such a way that $P$ is contained in $H_{F}^{+} \cup H_{F}$. By $F a c(P)$ we denote the set of all facets of $P$, by $\operatorname{Fac}_{H}(P)$ all hyperplanes coming from the facets, and by $\operatorname{Fac}_{H}^{+}(P)$ the set $\left\{H_{F}^{+} \mid F\right.$ facet of $\left.P\right\}$.

Further let $\operatorname{adj}(F)$ be the set of facets of $P$ adjacent to $F$. For a subset $\mathcal{F}$ of $\operatorname{adj}(F)$ we denote the set of hyperplanes defined by the facets in $\mathcal{F}$ by $\mathcal{F}_{H}$, and the set of positive half-spaces determined by the hyperplanes in $\mathcal{F}_{H}$ by $\mathcal{F}_{H}^{+}$.

Finally, if $v$ is some point in $\mathbb{R}^{d}$ then $v$ lies beyond a facet $F$ if $v \in H_{F}^{-}$and $v$ lies beneath $F$ if $v \in H_{F}^{+}$.

Let $S$ be a facet of a $d$-polytope $P$ and $\mathcal{F}, \mathcal{N}$ be disjoint subsets of $\operatorname{adj}(S)$. We define a set $\mathbf{N V}_{\mathcal{F}, \mathcal{N}}^{S}(P)$ by

$$
\mathbf{N V}_{\mathcal{F}, \mathcal{N}}^{S}(P):=\left(\bigcap_{H \in \mathcal{A}} H^{+}\right) \cap\left(\bigcap_{H \in \mathcal{F}_{H}} H\right) \cap\left(\bigcap_{H \in \mathcal{N}_{H} \cup\left\{H_{S}\right\}} H^{-}\right)
$$

for $\mathcal{A}:=\operatorname{Fac}_{H}(P) \backslash\left(\mathcal{F}_{H} \cup \mathcal{N}_{H} \cup\left\{H_{S}\right\}\right)$.

Definition 2.2 (Pseudo-Stacking). Let $P$ be a $d$-polytope and $S$ a simplex facet of $P$. Choose two disjoint sets $\mathcal{F}, \mathcal{N} \subseteq \operatorname{adj}(S)$. Assume that $\mathbf{N V}_{\mathcal{F}, \mathcal{N}}^{S}(P) \neq \emptyset$.

The pseudo-stacking $\mathbf{P S}_{\mathcal{F}, \mathcal{N}}^{S}(P)$ of $P$ above $S$ with respect to $\mathcal{F}$ and $\mathcal{N}$ is the convex hull $\operatorname{conv}(P \cup v)$ of $P$ with a point $v \in \mathbf{N V}_{\mathcal{F}, \mathcal{N}}^{S}(P)$. If $\mathcal{N}=\emptyset$ then we omit it in the notation and write $\mathbf{P S}_{\mathcal{F}}^{S}(P)$ for the pseudo-stacking of $P$ above $S$.

Compare this definition to Grünbaum Grü03 Section 5.2]. In plain words, a point $v \in \mathbf{N V}_{\mathcal{F}, \mathcal{N}}^{S}(P)$ lies beyond $S$ and all facet hyperplanes coming from facets in $\mathcal{N}$, it lies in all facet hyperplanes from facets in $\mathcal{F}$, and beneath all other facet hyperplanes. A priori, this set need not contain a point, but we will show some conditions that guarantee that this set is non-empty. See Figures 2.12.4 for some illustrations of this operation with various choices of $\mathcal{F}$ and $\mathcal{N}$.

We have the following simple fact about the pseudo-stacking operation:

Proposition 2.3. The combinatorial properties of $\mathbf{P S}_{\mathcal{F}, \mathcal{N}}^{S}(P)$ do not depend on the actual choice of the point $v \in \mathbf{N V}_{\mathcal{F}, \mathcal{N}}^{S}(P)$.

REMARK 2.4. The usual stacking operation is the special case $\mathcal{F}=\mathcal{N}=\emptyset$.

2.2. Properties of the Construction. Now we want to examine some cases in which the set $\mathbf{N} \mathbf{V}_{\mathcal{F}, \mathcal{N}}^{S}(P)$ is non-empty and thus pseudo-stacking can be applied. To simplify the statements of the propositions we introduce some more notation.

Definition 2.5. Let $P$ be a $d$-polytope and $F$ a facet of $P$. A subset $\mathcal{F}$ of $\operatorname{adj}(F)$ is called nonsimple if there is no pair of adjacent facets $G, G^{\prime} \in \mathcal{F}$ that have a common $(d-3)$-face with $F$.

Definition 2.6. Let $P$ be a $d$-polytope. A facet $F$ of $P$ is in bounded position if the hyperplanes in any subset of $\operatorname{adj}(F)_{H}$ of cardinality $d$ intersect in a point in $H_{F}^{-}$. Equivalently, $F$ is in bounded position if the intersection of the half-spaces defining $P$ remains bounded if we remove the half-space defined by $F$. 
See Figure 2.1 for an illustration of the applicability of our definition. The set $\mathcal{F}:=\left\{F_{1}, F_{2}\right\}$ of facets of the polytope $P$ with the chosen simplex facet $S$ in bounded position is nonsimple, while the set $\mathcal{F}^{\prime}:=\left\{F_{2}, F_{3}\right\}$ is not.

REMARK 2.7. There is always a projective transformation that puts a simplex facet $S$ of a $d$-polytope $P$ into bounded position, as long as there exists a facet of $P$ not adjacent to $S$, that is, if $P$ is not a simplex. Without loss of generality we can therefore assume that a simplex facet is in bounded position, if the given polytope itself is not a simplex.

In the following lemma we show that we can always apply our construction to a simplex facet in bounded position, regardless of the choice of $\mathcal{F}$ and $\mathcal{N}$.

Lemma 2.8. Let $P$ be a d-polytope and $S$ a simplex facet of $P$ in bounded position. Choose two disjoint sets $\mathcal{F}, \mathcal{N} \subseteq \operatorname{adj}(S)$. Then the set $\mathbf{N V}_{\mathcal{F}, \mathcal{N}}^{S}(P)$ is not empty.

Proof. Let $F_{1}, \ldots, F_{d}$ be the facets in $\operatorname{adj}(S)$ and number the vertices $p_{1}, \ldots, p_{d}$ of $S$ in such a way that $p_{j}$ is the unique vertex not lying in $F_{j}$ for $j=1, \ldots, d$.

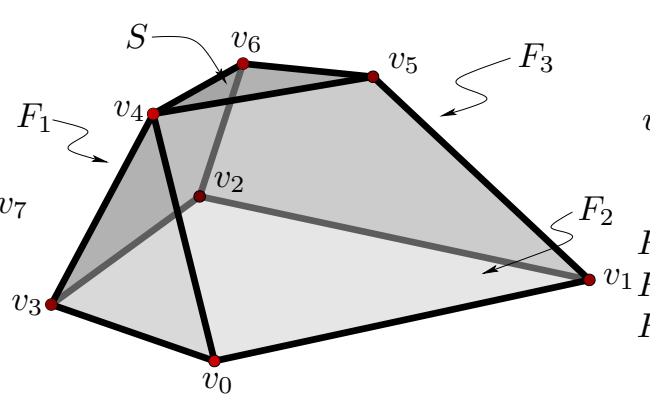

Figure 2.1. A polytope $P$ with simplex facet $S$ in bounded position and $\operatorname{adj}(S)=$ $\left\{F_{1}, F_{2}, F_{3}\right\}$.

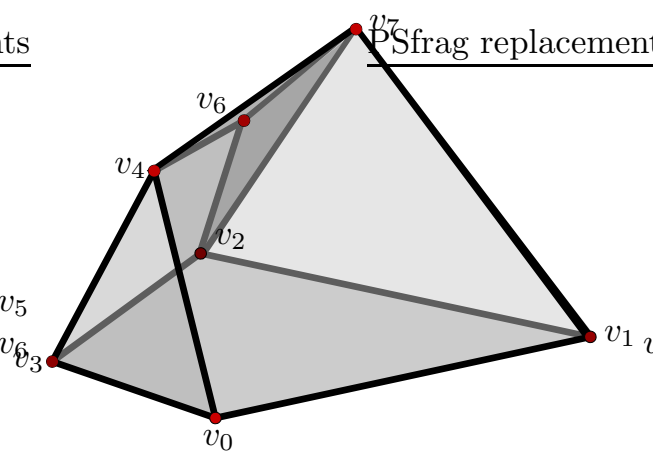

FiguRE

2.3. The polytope $\quad \mathbf{P S}_{\mathcal{F}, \mathcal{N}}^{S}(P)$ with $\mathcal{F}:=\left\{F_{2}\right\}$ and $\mathcal{N}:=\left\{F_{3}\right\}$.

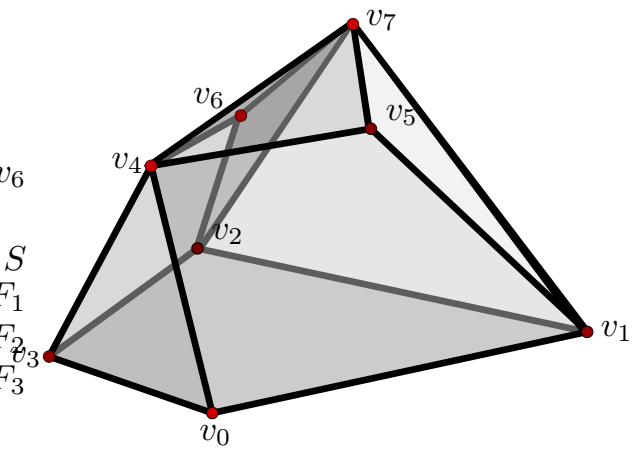

Figure 2.2. The polytope $\quad \mathbf{P S}_{\mathcal{F}, \mathcal{N}}^{S}(P)$ with $\mathcal{F}:=\emptyset$ and $\mathcal{N}:=$ $\left\{F_{3}\right\}$.

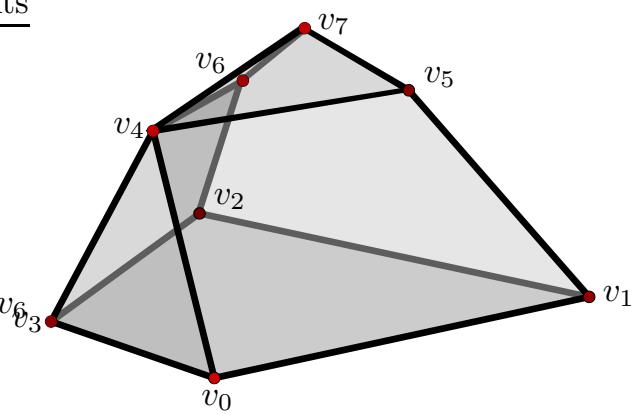

Figure 2.4. The polytope $\quad \mathbf{P S}_{\mathcal{F}, \mathcal{N}}^{S}(P)$ with $\mathcal{F}:=\left\{F_{3}\right\}$ and $\mathcal{N}:=\emptyset$. 
$S$ is a $(d-1)$-simplex and is in bounded position w.r.t. $P$, so all hyperplanes in the set $\operatorname{adj}(F)_{H}$ intersect in a unique point $p \in H_{S}^{-}$. Let $v_{j}:=p_{j}-p$ for $j=1, \ldots, d$. Define $p^{\prime} \in \mathbb{R}^{d}$ by

$$
p^{\prime}:=p+\varepsilon\left(\sum_{j: F_{j} \in \operatorname{adj}(S) \backslash(\mathcal{F} \cup \mathcal{N})} v_{j}-\sum_{j: F_{j} \in \mathcal{N}} v_{j}\right) .
$$

For $\varepsilon>0$ small enough $p^{\prime}$ is contained in $\mathbf{N V}_{\mathcal{F}, \mathcal{N}}^{S}(P)$.

The following proposition tells which types of facets occur in $\mathbf{P S}_{\mathcal{F}, \mathcal{N}}^{S}(P)$.

Proposition 2.9. Let $P$ be a d-polytope, $d \geq 3, S$ a simplex facet of $P$ in bounded position and $\mathcal{F}, \mathcal{N} \subseteq \operatorname{adj}(S), \mathcal{F} \cap \mathcal{N}=\emptyset$. Assume that $\mathcal{F}$ is nonsimple. Then the following properties of $\mathbf{P S}_{\mathcal{F}, \mathcal{N}}^{S}(P)$ hold:

(1) $S$ and the facets in $\mathcal{N}$ lie in the interior of $\mathbf{P S}_{\mathcal{F}, \mathcal{N}}^{S}(P)$.

(2) Let $v$ be the new vertex added by the pseudo-stacking. $\mathbf{P S}_{\mathcal{F}, \mathcal{N}}^{S}(P)$ has facets of the following four types:

(a) Facets not in $\{S\} \cup \mathcal{N} \cup \mathcal{F}$ remain unchanged in $\mathbf{P S}_{\mathcal{F}, \mathcal{N}}^{S}(P)$.

(b) For any ridge $R$ between $S$ and a facet in $\operatorname{adj}(S) \backslash(\mathcal{F} \cup \mathcal{N})$ we obtain a new facet that is a pyramid over $R$ with apex $v$.

(c) For any ridge $R$ between a facet in $\mathcal{N}$ and one in $\operatorname{Fac}(P) \backslash(\mathcal{F} \cup \mathcal{N} \cup\{S\})$ we obtain a new facet that is a pyramid over $R$ with apex $v$.

(d) For any facet $F \in \mathcal{F}$ we obtain a facet of the form $\mathbf{P S}_{\emptyset, \mathcal{N}_{F}}^{S \cap H_{F}}(F)$ in the pseudo-stacking, where we view $F$ as a polytope in $H_{F}$ and define $\mathcal{N}_{F}:=$ $\{N \cap F \mid N \in \mathcal{N}\}$.

Proof.

(1) $v$ lies beyond $S$ and all facets in $\mathcal{N}$ by definition. Hence, any ray from $v$ to a point $v^{\prime}$ in one of these facets intersects $P$ in a segment with boundary points $v^{\prime}$ and some other point $v^{\prime \prime}$. The segment from $v$ to $v^{\prime \prime}$ is contained in $\operatorname{conv}(P, v)$. Hence, $v^{\prime}$ must be in the interior of $\operatorname{conv}(P, v)$. This proves the first claim.

(2) The vertices of $\operatorname{conv}(P, v)$ are a subset of the vertices of $P$ and the vertex $v$. We show that all four facet types mentioned may occur in the pseudostacking, and no others.

(a) The new vertex $v$ lies beneath all facets not in $\mathcal{F} \cup \mathcal{N} \cup\{S\}$. Hence the facet hyperplanes of such facets remain valid and facet defining for the pseudo-stacking of $P$. This completely describes all facets of $\operatorname{conv}(P, v)$ that do not involve the vertex $v$.

(b) Let $F \notin \mathcal{F} \cup \mathcal{N}$ be a facet adjacent to $S$ and $R=F \cap S$. Then $v$ lies beneath $H_{F}$ and beyond $H_{S}$. Hence, any segment between $v$ and a point $v^{\prime} \in R$ intersects $P$ only in $v^{\prime}$; on the other hand, a segment between $v$ and some $v^{\prime \prime} \in F \backslash R$ must intersect the interior of $P$. In the convex hull of $v$ and $P$ we thus obtain a pyramid over $R$.

(c) As above, there is a segment from $v$ to any vertex of a ridge $R$ between a facet $N \in \mathcal{N}$ and a facet $F \in F a c(P) \backslash(\mathcal{F} \cup \mathcal{N} \cup\{S\})$, but not to any relative interior point of $F$. Hence, in the convex hull of $v$ and $P$ we again obtain a pyramid over $R$.

(d) Let $F$ be a facet in $\mathcal{F}$ and $G$ the ridge it shares with $S$. We look at $F$ as a polytope defined in the hyperplane $H_{F}$. The added vertex $v$ 
also lies in that hyperplane. $G$ is a facet of $F$. As $\mathcal{F}$ is nonsimple and $S$ a simplex facet, none of the facets of $F$ adjacent to $G$ is defined by a hyperplane coming from a facet in $\mathcal{F}$. Let $\mathcal{N}_{F}$ be the set of ridges $F$ shares with some facet of $\mathcal{N}$. This is a set of facets of $F$.

Hence, seen as a point in $H_{F}, v$ is beyond $G$, beyond all facets in $\mathcal{N}_{F}$ and beneath all other facet hyperplanes of $F$. So $F$ is pseudo-stacked by $v$ above $G$ and $\mathcal{N}_{F}$.

This completely describes all facets of $P$ that involve the vertex $v$. Hence we have described all possible facet types of $\operatorname{conv}(P, v)$.

Remark 2.10. Any simplex facet of $\mathbf{P S}_{\mathcal{F}, \mathcal{N}}^{S}(P)$ of type (b) or (c) in Proposition 2.9 is again in bounded position.

The following theorem tells which $k$-faces of $P$ are also $k$-faces of $\mathbf{P S}_{\mathcal{F}, \mathcal{N}}^{S}(P)$, for $0 \leq k \leq d-2$ (cf. also Grünbaum Grü03 Sec. 5.2]).

Theorem 2.11. Let $P$ be a d-polytope, $d \geq 3, S$ a simplex facet of $P$ in bounded position and $\mathcal{F}, \mathcal{N} \subseteq \operatorname{adj}(S), \mathcal{F} \cap \mathcal{N}=\emptyset$. Assume that $\mathcal{F}$ is nonsimple.

$A$-face $G$ of $P, 0 \leq k \leq d-2$ is again a $k$-face of $\mathbf{P S}_{\mathcal{F}, \mathcal{N}}^{S}(P)$ if and only if there is a facet $F \in F a c(P) \backslash(\mathcal{F} \cup \mathcal{N} \cup\{S\})$ that contains $G$.

Proof. If $G$ is contained in a facet $F \in \operatorname{Fac}(P) \backslash(\mathcal{F} \cup \mathcal{N} \cup\{S\})$, then $F$ is also a facet of $\mathbf{P S}_{\mathcal{F}, \mathcal{N}}^{S}(P)$ by Proposition 2.9 and $G$ is also a face of $\mathbf{P S}_{\mathcal{F}, \mathcal{N}}^{S}(P)$.

So assume that $G$ is a face of $P$ all of whose adjacent facets are contained in $\mathcal{A}:=\mathcal{F} \cup \mathcal{N} \cup\{S\}$. Then any ridge of $P$ containing $G$ is also only adjacent to facets in $\mathcal{A}$ and we can assume that $G$ is a ridge of $P$. Because $S$ is a simplex, $G$ must intersect $S$ in a subridge. Hence, by nonsimplicity, there is at most one facet from $\mathcal{F}$ adjacent to $G$.

(1) If there is no facet from $\mathcal{F}$ adjacent to $G$, then $v$ lies beyond both facet hyperplanes defining $G$. Hence, $G$ is in the interior of $\mathbf{P S}_{\mathcal{F}, \mathcal{N}}^{S}(P)$.

(2) If one of the facets adjacent to $G$ is $F \in \mathcal{F}$ and the other is $S$, then $F$ is stacked above $G$, hence $G$ vanishes in the interior of $F$.

(3) If one of the facets adjacent to $G$ is $F \in \mathcal{F}$ and the other facet is in $\mathcal{N}$, then the pseudo-stacking transforms $F$ into the facet $\mathbf{P S}_{\emptyset, \mathcal{N}^{\prime}}^{S \cap F}(F)$, where $\mathcal{N}^{\prime}:=\{N \cap F \mid N \in \mathcal{N}\}$ and $G$ is one of the facets in $\mathcal{N}^{\prime}$ which vanish in the pseudo-stacking.

Restricting to the vertices of $P$ we have the following consequence.

Corollary 2.12. Let $P$ be a d-polytope, $d \geq 3, S$ a simplex facet of $P$ in bounded position and $\mathcal{F}, \mathcal{N} \subseteq \operatorname{adj}(S), \mathcal{F} \cap \mathcal{N}=\emptyset$. Assume that $\mathcal{F}$ is nonsimple and that any vertex of $P$ is contained in at least one facet $F \in F a c(P) \backslash(\mathcal{F} \cup \mathcal{N} \cup\{S\})$. Then $f_{0}\left(\mathbf{P S}_{\mathcal{F}, \mathcal{N}}^{S}(P)\right)=f_{0}(P)+1$.

Proof. By the previous theorem all vertices of $P$ are also vertices of $\mathbf{P S}_{\mathcal{F}, \mathcal{N}}^{S}(P)$. The pseudo-stacking operation adds one new vertex.

The next theorems deal with the consequences of the pseudo-stacking operation on $k$-simpliciality and $h$-simplicity.

TheOREM 2.13. Let $P$ be a $k$-simplicial $d$-polytope for $d \geq 3$ and $1 \leq k \leq d-2$ with a simplex facet $S$ in bounded position. Let $\mathcal{F}, \mathcal{N} \subseteq \operatorname{adj}(S)$ be disjoint sets. Assume that $\mathcal{F}$ is nonsimple. Then $\mathbf{P S}_{\mathcal{F}, \mathcal{N}}^{S}(P)$ is k-simplicial. 
Proof. Let $v$ be the added vertex in the pseudo-stacking. We use the characterisation of the facets in Proposition 2.9 The $k$-faces in facets that stay unchanged clearly stay combinatorially equivalent to a $k$-simplex. The $k$-faces contained in facets that are pyramids over ridges of $P$ are either already faces of $P$ or pyramids over $(k-1)$-faces of $P$ with apex $v$. Hence they are simplices.

All remaining facets are obtained by pseudo-stacking a facet of $P$. However, only the case $\mathcal{F}=\emptyset$ occurs, that is, in the pseudo-stacking of a facet none of its facets gets stacked. Therefore, the added facets are all pyramids over ridges, which preserve $k$-simpliciality for $k \leq d-2$.

Now we look more closely at the types of edges that can occur in $\operatorname{PS}_{\mathcal{F}, \mathcal{N}}^{S}(P)$ and determine their number. This is used to establish 2 -simplicity for the family of polytopes we construct in the next section. The two cases $\mathcal{N}=\emptyset$ and $\mathcal{N} \neq \emptyset$ are treated separately in the following two propositions, and the latter case is further restricted to $|\mathcal{N}|=1$.

Definition 2.14. Let $P$ be a polytope. For any face $F$ of $P$ let $\operatorname{fdeg}_{P}(F)$ be the number of facets of $P$ adjacent to $F$.

Proposition 2.15. Let $P$ be a d-polytope, $d \geq 3, S$ a simplex facet of $P$ in bounded position, $\mathcal{F} \subseteq \operatorname{adj}(S)$ nonsimple and $v \in \mathbf{N V}_{\mathcal{F}, \emptyset}^{S}(P)$.

(1) All edges of $\mathbf{P S}_{\mathcal{F}}^{S}(P)$ are of one of the following two types:

(a) edges e in $P$ such that $e \subset F$ for a facet $F \in F a c(P) \backslash(\{S\} \cup \mathcal{F})$;

(b) edges $e=\left[v, v^{\prime}\right]$ for every vertex $v^{\prime} \in S$; in this case

$$
\operatorname{fdeg}_{\mathbf{P S}}(P)(e)=\operatorname{fdeg}_{S}\left(v^{\prime}\right) .
$$

(2) $f_{1}\left(\mathbf{P S}_{\mathcal{F}}^{S}(P)\right)=f_{1}(P)+d-f$, where $f=|\mathcal{F}|$ if $d=3$ and $f=0$ otherwise.

PROOF.

(1) The edges of $\mathbf{P S}_{\mathcal{F}}^{S}(P)$ that are also edges of $P$ are exactly the ones described in (a), by Theorem 2.11 On the other hand, if an edge $e$ of $\mathbf{P S}_{\mathcal{F}}^{S}(P)$ is not an edge of $P$, then one of its vertices is $v$ and the other vertex $v^{\prime}$ is contained in $S$, which is the only facet of $P$ whose vertices can be seen by $v$. In this case, every facet of $\mathbf{P S}_{\mathcal{F}}^{S}(P)$ that contains $e$ corresponds to a ridge $R \subset S$ such that $v^{\prime} \in R$ - either the facet is of type (b) or of type (d) in Proposition 2.9

(2) Since $S$ is a simplex there are $d$ edges of type (b). If $d>3$ then, since $\mathcal{F}$ is nonsimple, every edge of $P$ is contained in at least one facet not in $\mathcal{F} \cup\{S\}$, hence there are $f_{1}(P)$ edges of type (a). If $d=3$, then edges are ridges and every ridge $F \cap S$ for $F \in \mathcal{F}$ disappears - namely, it is the base facet for the pseudo-stacking of $F$ (cf. Proposition 2.9). In this case we have $f_{1}(P)-|\mathcal{F}|$ edges of type (a).

Proposition 2.16. Let $P$ be a d-polytope, $d \geq 3, S$ a simplex facet of $P$ in bounded position, $\mathcal{F} \subseteq \operatorname{adj}(S)$ nonsimple, $\mathcal{N}=\{N\}$ with a facet $N \in \operatorname{adj}(S) \backslash \mathcal{F}$ and $v \in \mathbf{N V}_{\mathcal{F}, \mathcal{N}}^{S}(P)$. Additionally, suppose that every vertex of $S$ and $N$ is contained in at least one facet from $F a c(P) \backslash(\{S\} \cup \mathcal{F} \cup \mathcal{N})$.

(1) All edges of $\mathbf{P S}_{\mathcal{F}, \mathcal{N}}^{S}(P)$ are of one of the following types:

(a) edges e in $P$ such that $e \subset F$ for a facet $F \in F a c(P) \backslash(\{S\} \cup \mathcal{F} \cup \mathcal{N})$; 
(b) edges $e=\left[v, v^{\prime}\right]$ for every vertex $v^{\prime}$ of either $S$ or $N$; in this case

$$
\operatorname{fdeg}_{\mathbf{P} \mathbf{S}_{\mathcal{F}, \mathcal{N}}^{S}(P)}(e)=\operatorname{fdeg}_{X}\left(v^{\prime}\right),
$$

where $X=S$ or $X=N$ respectively;

(c) edges $e=\left[v, v^{\prime}\right]$ for every vertex $v^{\prime}$ of $S \cap N$.

(2) $f_{1}\left(\mathbf{P S}_{\mathcal{F}, \mathcal{N}}^{S}(P)\right)=f_{1}(P)+d+f_{0}(N)-f_{0}(S \cap N)-f$, where $f=|\mathcal{F}|$ if $d=3$ and $f=0$ otherwise.

ProOF.

(1) The description of types of edges is complete by the same argument as in the proof before; the only difference is that here the vertex $v$ sees both facets $S$ and $N$. Also, if $v^{\prime}$ is a vertex in either $S$ or $N$, the edge $\left[v, v^{\prime}\right]$ is contained in the facets corresponding to the ridges of $S$, resp. $N$, that are adjacent to $v^{\prime}$.

(2) Again, since $S$ is a simplex, $\mathbf{P S}_{\mathcal{F}, \mathcal{N}}^{S}(P)$ has $d+f_{0}(N)-f_{0}(S \cap N)$ edges of type (b) or (c). If $d>3$ then $\mathcal{F}$ being nonsimple ensures that every edge of $P$ is also an edge of $\mathbf{P S}_{\mathcal{F}, \mathcal{N}}^{S}(P)$ of type (a). If $d=3$, again all edges between facets in $\mathcal{F}$ and $S$ or $N$ disappear; there is no facet in $\mathcal{F}$ that shares ridges with both $S$ and $N$, since if there were, we had a vertex contained only in facets from $\{S\} \cup \mathcal{F} \cup \mathcal{N}$. Therefore exactly $f$ edges from $P$ are no edges in $\mathbf{P S}_{\mathcal{F}, \mathcal{N}}^{S}(P)$.

We want to show in the next section that certain combinations of pseudo-stacking operations preserve 2-simplicity of a 4 -polytope $P$. For this we have to count the number of facets a subridge of $P$ is in. We do this with the following two propositions. Again, we consider the two cases $\mathcal{N}=\emptyset$ and $\mathcal{N} \neq \emptyset$ separately and restrict the latter to $|\mathcal{N}|=1$.

Proposition 2.17. Let $P$ be a d-polytope, $d \geq 3, S$ a simplex facet of $P$ in bounded position, $\mathcal{F} \subseteq \operatorname{adj}(S)$ nonsimple and $G$ a subridge of $P$ with $G \subset S$. Define $\varphi:=|\{F \in \mathcal{F} \mid G \subset F\}|$.

Then $0 \leq \varphi \leq 2$ and $G$ is a face of $\mathbf{P S}_{\mathcal{F}}^{S}(P)$; furthermore,

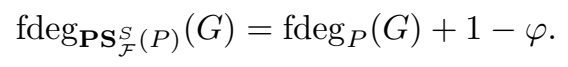

Proof. $G$ is a ridge of $S$, hence there are two facets $G_{1}, G_{2}$ of $S$ such that $G_{1} \cap G_{2}=G$. Since $G_{1}, G_{2}$ are ridges of $P$, if $G$ is contained in some facet $F \in \mathcal{F}$, then $F \cap S \in\left\{G_{1}, G_{2}\right\}$. Therefore at most 2 facets in $\mathcal{F}$ can possibly contain $G$.

If $G$ was not a face of $\mathbf{P S}_{\mathcal{F}}^{S}(P)$ then by Theorem 2.11 it would be contained in two facets $F_{1}, F_{2} \in \mathcal{F}$. Since $S$ is a simplex, $F_{1} \cap F_{2}$ was a ridge containing $G$, in contradiction to $\mathcal{F}$ being nonsimple.

Now count the number of facets of $\mathbf{P S}_{\mathcal{F}}^{S}(P)$ containing $G$. All facets of $P$ not in $\mathcal{F} \cup\{S\}$ remain facets of $\mathbf{P S}_{\mathcal{F}}^{S}(P)$ by Proposition 2.9 therefore, $\operatorname{fdeg}_{P}(G)-\varphi-1$ facets still contain $G$ in $\operatorname{PS}_{\mathcal{F}}^{S}(P)$. All facets in $\mathcal{F}$ containing $G$ also stay facets of $\mathbf{P S}_{\mathcal{F}}^{S}(P)$ containing $G$. Additionally, for every one of the two ridges $R$ of $P$ with $G \subset R \subset S$, we either pseudo-stacked the facet $F$ with $F \cap S=R$ (if it is in $\mathcal{F}$ ) or we get a new facet, which is a pyramid over $R$; in the latter case, we have not counted it yet.

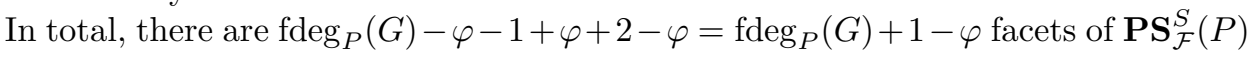
that contain $G$. 
Proposition 2.18. Let $P, S, \mathcal{F}, G$ and $\varphi$ as in Proposition 2.17. Choose a facet $N \in \operatorname{adj}(S) \backslash \mathcal{F}$ and set $\mathcal{N}=\{N\}$.

(1) If $G \subset S$ and $G \not \subset N$, then $G$ is a face of $\mathbf{P S}_{\mathcal{F}, \mathcal{N}}^{S}(P)$ and

$$
\operatorname{fdeg}_{\mathbf{P S}_{\mathcal{F}, \mathcal{N}}^{S}(P)}(G)=\operatorname{fdeg}_{P}(G)+1-\varphi .
$$

(2) If $G \subset N$ and every facet adjacent to $G$ is either $S, N$ or in $\mathcal{F}$, then $G$ is not a face of $\mathbf{P} \mathbf{S}_{\mathcal{F}, \mathcal{N}}^{S}(P)$; otherwise, $G$ is a face of $\mathbf{P S}_{\mathcal{F}, \mathcal{N}}^{S}(P)$ and

$$
\operatorname{fdeg}_{\mathbf{P S} \mathbf{S}_{\mathcal{F}, \mathcal{N}}^{S}(P)}(G)=\operatorname{fdeg}_{P}(G)+1-\varepsilon-\varphi
$$

where $\varepsilon=1$ if $G \subset S$ and $\varepsilon=0$ if not.

Proof. (1) can be shown in the same way as Proposition 2.17 since the facet $N$ has no influence whatsoever on $G$.

Suppose $G$ is contained in $N$. By Theorem 2.11 $G$ is a face of $\mathbf{P S}_{\mathcal{F}, \mathcal{N}}^{S}(P)$ if and only if there is facet adjacent to $G$ that is not in $\mathcal{F} \cup\{S, N\}$. In this case, the number of facets of $\mathbf{P S}_{\mathcal{F}, \mathcal{N}}^{S}(P)$ can be computed in the same way as in the previous proof, except that if $G \subset S$, an additional facet containing $G$ (namely $S$ ) disappears.

REMARK 2.19. The construction given in this section can be further generalised:

(1) Nonsimplicity of the set $\mathcal{F}$ is not necessary.

(2) The facet $S$ need not be a simplex.

(3) In this case the construction can be adapted to only apply to a part of $S$. Several new families of polytopes arise via these generalisations. However, keeping track of the changes in the $f$-vector is more subtle in these cases. We do not need them here, so we omit their treatment.

\section{Generating 2-simple and 2-simplicial 4-polytopes}

In this part we use the pseudo-stacking operation defined in the previous section to extend elementary 2-simple and 2-simplicial 4-polytopes while maintaining these properties. As we have seen in Theorem 2.13 pseudo-stacking preserves 2simpliciality. The more difficult part is to also preserve 2-simplicity; we accomplish this by applying the operation five, resp. four times in a suitable way, such that edges whose degree initially increases are again contained in only 3 facets at the end.

To simplify the task of keeping track of the facets generated in each step of the construction we introduce two ways of distinguishing certain facets.

Definition 3.1. Let $P$ be a $d$-polytope and $S$ a facet of $P$.

(1) If the vertices $v_{0}, \ldots, v_{k}$ all lie in one facet of $P$ and define this facet uniquely, then we denote this facet by $F\left(v_{0}, \ldots, v_{k}\right)$.

(2) If $e_{0}, \ldots, e_{m}$ are edges of a facet $G$ of $S$, then we denote the unique facet of $P$ adjacent to $S$ via $G$ by $F_{S}\left(e_{0}, \ldots, e_{m}\right)$.

3.1. The first construction. Let $P$ be a 4-polytope and $S$ a simplex facet of $P$ in bounded position. $S$ has six edges and four vertices, which we label $e_{0}, e_{1}, \ldots, e_{5}$ and $v_{0}, v_{1}, v_{2}, v_{3}$ according to Figure 1(a)

We proceed with the five pseudo-stacking steps described in Table 3.1. In Step $i$ we construct the polytope $P^{(i)}:=\mathbf{P S}_{\mathcal{F}_{i}}^{S_{i}}\left(P^{(i-1)}\right)$, with $P^{(0)}:=P$ and the parameters as given in Table 3.1 For every step we additionally give a complete list of facets 


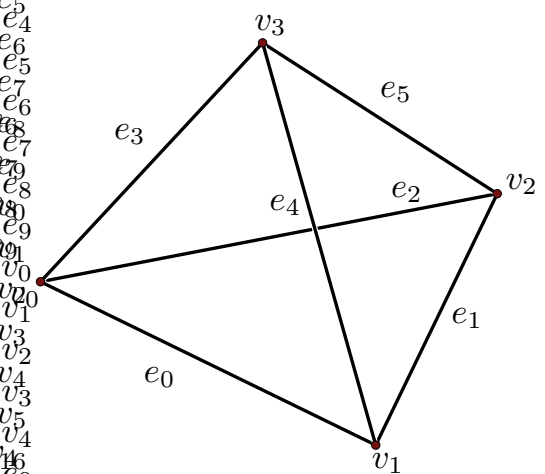

(a) Labelling of the vertices and edges of the simplex $S$.

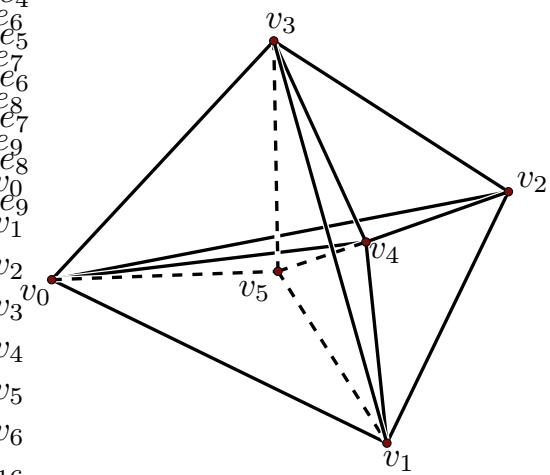

(c) Part of the Schlegel diagram of $P^{(2)}$; note that $v_{1} \ldots, v_{5}$ define a bipyramid over a triangle, which occurred by stacking the facet $F\left(v_{1}, v_{2}, v_{3}, v_{4}\right)$ of $P^{(1)}$.

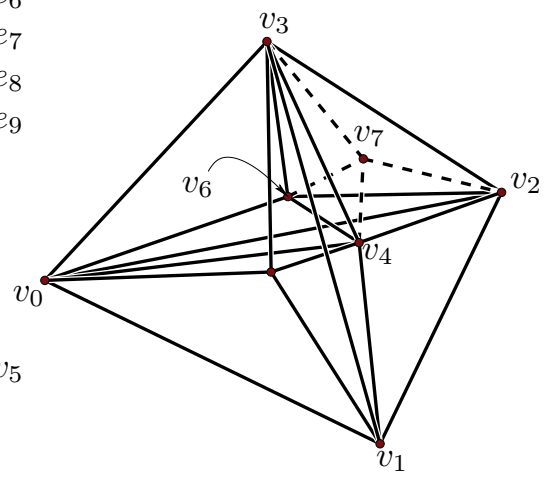

(e) Part of the Schlegel diagram of $P^{(4)}$ showing the pseudo-stacking of the facet $F\left(v_{2}, v_{3}, v_{4}, v_{6}\right)$ of $P^{(3)}$.

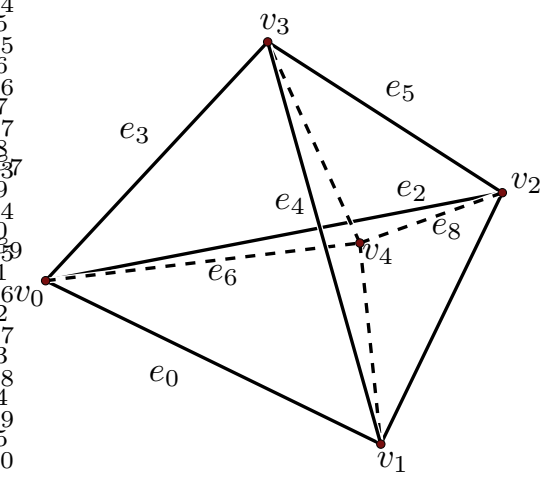

(b) Part of the Schlegel diagram of $P^{(1)}$; the new edges $e_{6}, \ldots, e_{9}$ (partly labelled) are drawn as dashed lines.

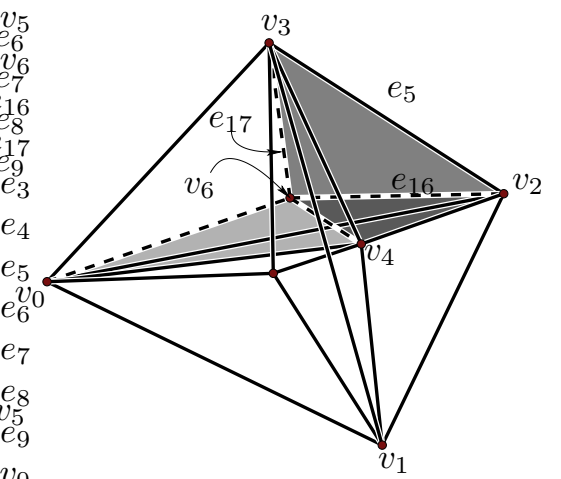

(d) Part of the Schlegel diagram of $P^{(3)}$; the shaded facets are the base facets for the last two pseudo-stacking steps.

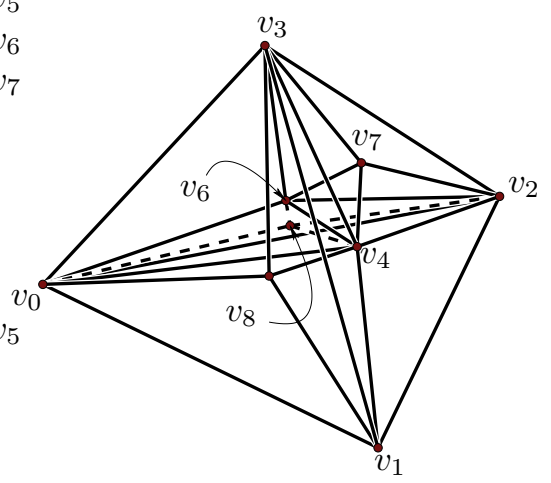

(f) Part of the Schlegel diagram of $P^{(5)}$ showing the pseudo-stacking of the facet $F\left(v_{0}, v_{2}, v_{4}, v_{6}\right)$ of $P^{(4)}$.

FiguRE 3.1. The first construction. 
TABLE 3.1. Summary of parameters and involved faces for the first construction.

\begin{tabular}{|c|c|c|c|c|c|}
\hline step $i$ & base facet $S_{i}$ & facets in $\mathcal{F}_{i}$ & new facets & changing edges & degree \\
\hline 1 & $S$ & $F_{S}\left(e_{0}, e_{1}, e_{2}\right)$ & $\overline{F\left(v_{0}, v_{1}, v_{3}, v_{4}\right)}$ & $e_{6}:=\left[v_{0}, v_{4}\right]$ & 3 \\
\hline \multirow[t]{6}{*}{ (Fig. 1(b) } & & & $F\left(v_{1}, v_{2}, v_{3}, v_{4}\right)$ & $e_{7}:=\left[v_{1}, v_{4}\right]$ & 3 \\
\hline & & & $F\left(v_{0}, v_{2}, v_{3}, v_{4}\right)$ & $e_{8}:=\left[v_{2}, v_{4}\right]$ & 3 \\
\hline & & & & $e_{9}:=\left[v_{2}, v_{4}\right]$ & 3 \\
\hline & & & & $e_{3}$ & $d_{3}+1$ \\
\hline & & & & $e_{4}$ & $d_{4}+1$ \\
\hline & & & & $e_{5}$ & $d_{5}+1$ \\
\hline \multirow{6}{*}{$\begin{array}{c}2 \\
\text { (Fig. } 1(\mathrm{c})\end{array}$} & $F\left(v_{0}, v_{1}, v_{3}, v_{4}\right)$ & $F\left(v_{1}, v_{2}, v_{3}, v_{4}\right)$ & $F\left(v_{0}, v_{3}, v_{4}, v_{5}\right)$ & {$\left[v_{0}, v_{5}\right]$} & 3 \\
\hline & & $F_{S_{2}}\left(e_{0}, e_{3}, e_{4}\right)$ & $F\left(v_{0}, v_{1}, v_{4}, v_{5}\right)$ & {$\left[v_{1}, v_{5}\right]$} & 3 \\
\hline & & & & {$\left[v_{3}, v_{5}\right]$} & 3 \\
\hline & & & & {$\left[v_{4}, v_{5}\right]$} & 3 \\
\hline & & & & $e_{4}$ & $d_{4}$ \\
\hline & & & & $e_{6}$ & 4 \\
\hline \multirow{6}{*}{$\begin{array}{c}3 \\
\text { (Fig. } 1(\mathrm{~d})\end{array}$} & $F\left(v_{0}, v_{2}, v_{3}, v_{4}\right)$ & $F\left(v_{0}, v_{3}, v_{4}, v_{5}\right)$ & $F\left(v_{2}, v_{3}, v_{4}, v_{6}\right)$ & $e_{15}:=\left[v_{0}, v_{6}\right]$ & 3 \\
\hline & & $F_{S_{3}}\left(e_{2}, e_{3}, e_{5}\right)$ & $F\left(v_{0}, v_{2}, v_{4}, v_{6}\right)$ & $e_{16}:=\left[v_{2}, v_{6}\right]$ & 3 \\
\hline & & & & $e_{17}:=\left[v_{3}, v_{6}\right]$ & 3 \\
\hline & & & & $e_{18}:=\left[v_{4}, v_{6}\right]$ & 3 \\
\hline & & & & $e_{3}$ & $d_{3}$ \\
\hline & & & & $e_{8}$ & 4 \\
\hline \multirow{6}{*}{$\begin{array}{c}4 \\
\text { (Fig. } 1(\mathrm{e})\end{array}$} & $F\left(v_{2}, v_{3}, v_{4}, v_{6}\right)$ & $F\left(v_{1}, v_{2}, v_{3}, v_{4}\right)$ & $F\left(v_{3}, v_{4}, v_{6}, v_{7}\right)$ & $\overline{\left[v_{2}, v_{7}\right]}$ & 3 \\
\hline & & $F_{S_{4}}\left(e_{5}, e_{16}, e_{17}\right)$ & $F\left(v_{2}, v_{4}, v_{6}, v_{7}\right)$ & {$\left[v_{3}, v_{7}\right]$} & 3 \\
\hline & & & & {$\left[v_{4}, v_{7}\right]$} & 3 \\
\hline & & & & {$\left[v_{6}, v_{7}\right]$} & 3 \\
\hline & & & & $e_{5}$ & $d_{5}$ \\
\hline & & & & $e_{18}$ & 4 \\
\hline \multirow{7}{*}{$\begin{array}{c}5 \\
\text { (Fig. } 1(\mathrm{f})\end{array}$} & $F\left(v_{0}, v_{2}, v_{4}, v_{6}\right)$ & $F\left(v_{0}, v_{3}, v_{4}, v_{5}, v_{6}\right)$ & $F\left(v_{0}, v_{2}, v_{6}, v_{8}\right)$ & {$\left[v_{0}, v_{8}\right]$} & 3 \\
\hline & & $F\left(v_{2}, v_{4}, v_{6}, v_{7}\right)$ & & {$\left[v_{2}, v_{8}\right]$} & 3 \\
\hline & & $F_{S_{5}}\left(e_{2}, e_{6}, e_{8}\right)$ & & {$\left[v_{4}, v_{8}\right]$} & 3 \\
\hline & & & & {$\left[v_{6}, v_{8}\right]$} & 3 \\
\hline & & & & $e_{6}$ & 3 \\
\hline & & & & $e_{8}$ & 3 \\
\hline & & & & $e_{18}$ & 3 \\
\hline
\end{tabular}

of type (b) in Proposition 2.9 as well as of edges that either show up by Proposition 2.15] or change their degree by Proposition 2.17 note that the only edges of $P$ that change degree during the process are $e_{3}, e_{4}$ and $e_{5}$, whose degrees in $P$ are denoted by $d_{3}, d_{4}$ and $d_{5}$ respectively.

Due to Remark [2.10] the base facets in each step are in bounded position; additionally, it can be verified easily that the sets $\mathcal{F}_{i}$ are nonsimple.

Definition 3.2. Let $P$ be a 4 -polytope with a simplex facet $S$ in bounded position. We denote the polytope $P^{(5)}$ obtained by applying the five steps of Table 3.1 by $\mathcal{I}^{1}(P ; S)$.

REMARK 3.3. Note that $\mathcal{I}^{1}(P ; S)$ implicitly depends on a labelling of the vertices of $S$. Choosing different labellings may result in combinatorially different polytopes for the same choice of $P$ and $S$.

Lemma 3.4. Let $P$ be a 4-polytope with a simplex facet $S$ in bounded position and the vertices of $S$ numbered in arbitrary order. Then $\mathcal{I}^{1}(P ; S)$ has again simplex facets in bounded position. Additionally, all edges of $P$ are still present in $\mathcal{I}^{1}(P ; S)$, their degrees remain unchanged and all new edges have degree 3. 


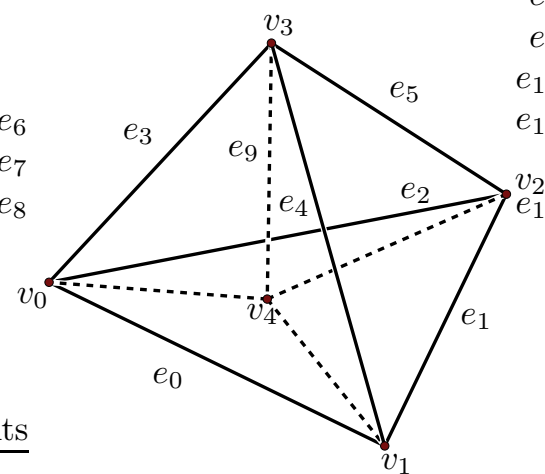

(a) Part of the Schlegel diagram of $Q^{(1)}$ with the labelling inherited from the facet $S$ of $Q$.

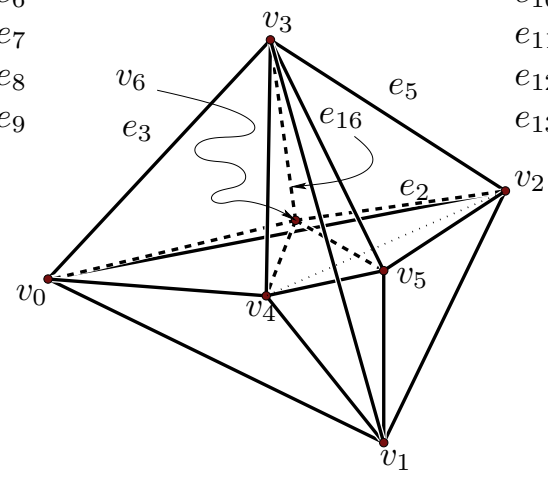

(c) Part of the Schlegel diagram of $Q^{(3)}$ where the edge $e_{7}$ (indicated as a dotted line) from $Q^{(2)}$ is no longer an edge.

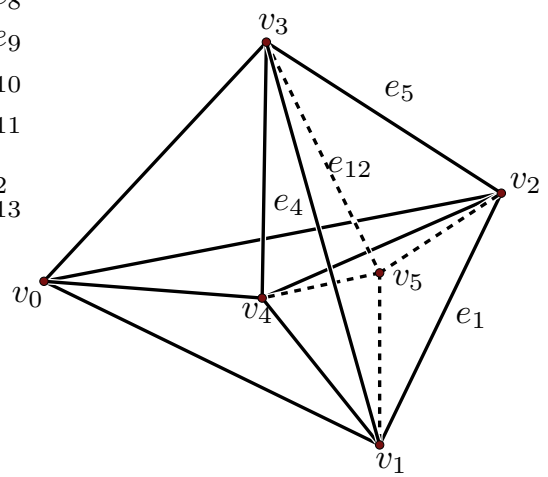

(b) Part of the Schlegel diagram of $Q^{(2)}$, where the dashed edges were obtained by pseudo-stacking the facet $F\left(v_{1}, v_{2}, v_{3}, v_{4}\right)$ from the previous step.

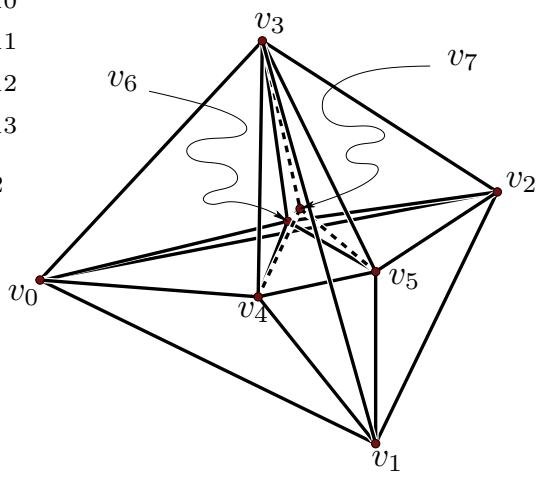

(d) Part of the Schlegel diagram of $Q^{(4)}$ with the simplex facet $F\left(v_{4}, v_{5}, v_{6}, v_{7}\right)$ obtained in the last step.

Figure 3.2. The second construction.

3.2. A second construction. Let $Q$ be a 4 -polytope with a simplex facet $S$ in bounded position. We label the vertices and edges of $S$ in the same way as in the previous section (see Figure 1(a).

The construction is described by Table 3.2 in the same way as before. The main difference is that in Step 3 the set $\mathcal{N}_{3}$ is not empty, so in general we construct $Q^{(i)}:=\mathbf{P S}_{\mathcal{F}_{i}, \mathcal{N}_{i}}^{S_{i}}\left(Q^{(i-1)}\right)$ with the given parameters. Note that one effect of this is that in Step 3 one edge disappears by Theorem 2.11] also, the only edges of $Q$ changing their degree are $e_{1}, e_{2}$ and $e_{5}$, whose degrees are labelled accordingly.

Definition 3.5. Let $Q$ be a 4-polytope with a simplex facet $S$ in bounded position. We denote the polytope $Q^{(4)}$ obtained by applying the four steps of Table 3.2 by $\mathcal{I}^{2}(Q ; S)$. 


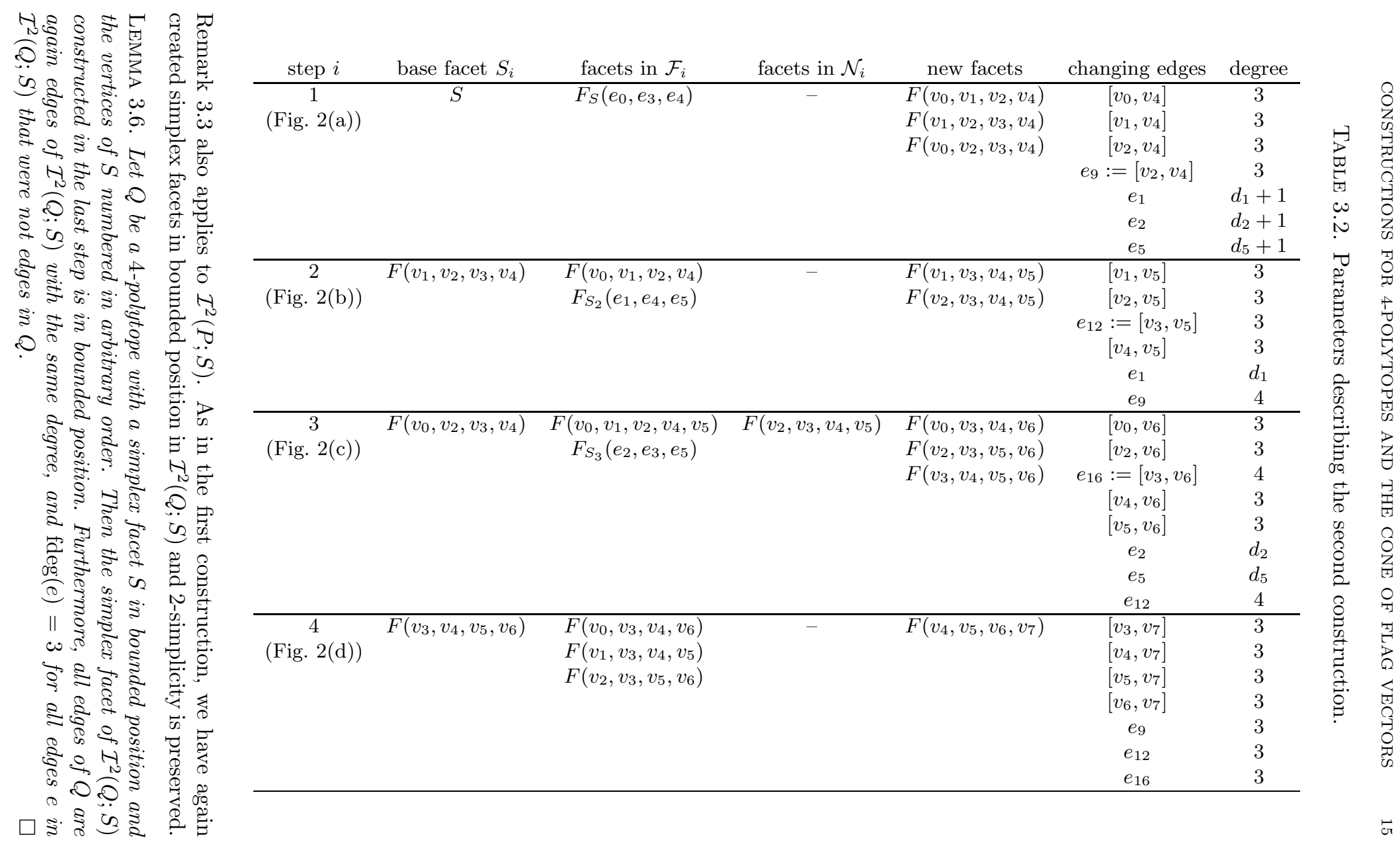


3.3. Properties of the Constructions. The two constructions preserve the properties we are interested in.

TheOrem 3.7. Let $P$ be a 4-polytope with a simplex facet $S$ in bounded position. Then

$$
f\left(\mathcal{I}^{1}(P ; S)\right)=f(P)+(5,20,20,5),
$$

and

$$
f\left(\mathcal{I}^{2}(P ; S)\right)=f(P)+(4,16,16,4) .
$$

Proof. In every step of both constructions, we add one vertex each by Corollary 2.12 Hence $f_{0}\left(\mathcal{I}^{1}(P ; S)\right)=f_{0}(P)+5$ and $f_{0}\left(\mathcal{I}^{2}(P ; S)\right)=f_{0}(P)+4$.

Propositions 2.15 and 2.16 tell how many edges are added in each step. Accordingly, the first construction adds 4 edges in each step. In Steps 1, 2 and 4 of the second construction, we also add 4 edges each; in the third step 5 edges are added, but one edge is destroyed, so the overall change is also 4 . Therefore, we have $f_{1}\left(\mathcal{I}^{1}(P ; S)\right)=$ $f_{1}(P)+5 \cdot 4=f_{1}(P)+20$ and $f_{1}\left(\mathcal{I}^{2}(P ; S)\right)=f_{1}(P)+4 \cdot 4=f_{1}(P)+16$. Finally, the number of new facets in the respective steps can be read of from Tables 3.1 and 3.2 (or Proposition 2.9) - note that the facets $S_{i}$ in every step, as well as the facet $Q^{(3)}$ in $\mathcal{N}_{3}$, disappear. Summarising, we get $f_{3}\left(\mathcal{I}^{1}(P ; S)\right)=f_{3}(P)+5$ and $f_{3}\left(\mathcal{I}^{2}(P ; S)\right)=f_{3}(P)+4$.

By Euler's equation, the number of ridges is determined by $f_{0}, f_{1}$ and $f_{3}$, which implies the claim.

COROLlary 3.8. Let $P$ be a 4-polytope with a simplex facet $S$ in bounded position. Then $g_{2}\left(\mathcal{I}^{1}(P ; S)\right)=g_{2}\left(\mathcal{I}^{2}(P ; S)\right)=g_{2}(P)$.

Proof. Define $a(P):=f_{02}(P)-3 f_{2}(P)$ and $b(P):=f_{1}(P)-4 f_{0}(P)$. Then $g_{2}(P)=a(P)+b(P)+10$.

We first show that $a\left(\mathcal{I}^{1}(P ; S)\right)=a(P)$. Any 2 -face of $\mathcal{I}^{1}(P ; S)$ that is not a face of $P$ is a triangle by Proposition 2.9 Furthermore, any 2-face of $P$ that is not a face of $\mathcal{I}^{1}(P ; S)$ is a ridge of the base facet in one of the five steps; since all base facets are simplices, all such 2 -faces are also triangles. Therefore, none of these faces causes a change in $a(P)$.

For $b(P)$, we have, by Theorem 3.7

$$
\begin{aligned}
b\left(\mathcal{I}^{1}(P ; S)\right) & =f_{1}\left(\mathcal{I}^{1}(P ; S)\right)-4 f_{0}\left(\mathcal{I}^{1}(P ; S)\right) \\
& =f_{1}(P)+20-4\left(f_{0}(P)+5\right)=f_{1}(P)-4 f_{0}(P)=b(P) .
\end{aligned}
$$

This implies $g_{2}\left(\mathcal{I}^{1}(P ; S)\right)=g_{2}(P)$.

Similar reasoning shows the claim for $\mathcal{I}^{2}(P ; S)$.

TheOREM 3.9. Let $P$ be a 2-simplicial, 2-simple 4-polytope with a simplex facet $S$ in bounded position. Then $\mathcal{I}^{1}(P ; S)$ and $\mathcal{I}^{2}(P ; S)$ are again 2-simple and 2-simplicial.

Proof. By Theorem 2.13 all intermediate polytopes in both constructions are 2-simplicial, hence also $\mathcal{I}^{1}(P ; S)$ and $\mathcal{I}^{2}(P ; S)$. Furthermore, all edges in $\mathcal{I}^{1}(P ; S)$ and $\mathcal{I}^{2}(P ; S)$ have degree 3 by Lemmas 3.4 and 3.6 and since this is true for $P$.

REMARK 3.10. There are more sequences of pseudo-stacking operations that preserve $g_{2}=0$ than the two given here. Even more can be constructed with the help of a further generalised pseudo-stacking operation, see Remark 2.19 


$$
\begin{aligned}
& \left\{v_{1}, v_{2}, v_{3}, v_{4}, v_{5}\right\} \\
& \left\{v_{3}, v_{4}, v_{5}, v_{7}, v_{8}\right\} \\
& \left\{v_{5}, v_{6}, v_{7}, v_{8}\right\} \\
& \left\{v_{2}, v_{4}, v_{5}, v_{6}, v_{8}\right\} \\
& \left\{v_{0}, v_{1}, v_{2}, v_{3}\right\} \\
& \left\{v_{0}, v_{2}, v_{3}, v_{5}, v_{6}, v_{7}\right\} \\
& \left\{v_{0}, v_{4}, v_{6}, v_{7}, v_{8}\right\} \\
& \left\{v_{0}, v_{1}, v_{3}, v_{4}, v_{7}\right\} \\
& \left\{v_{0}, v_{1}, v_{2}, v_{4}, v_{6}\right\}
\end{aligned}
$$

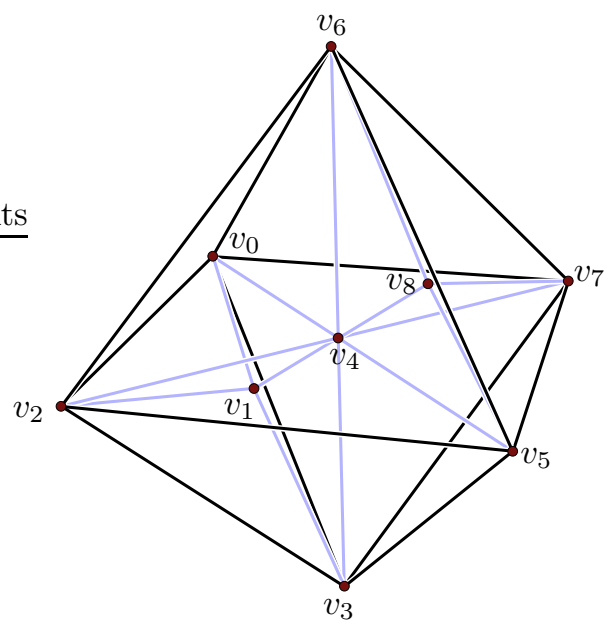

Figure 4.1. The vertex-facet-incidences and a Schlegel diagram of the polytope $W_{9}$.

A more symmetric version using these generalisations also applies in dimensions $d \geq$ 5 , i.e. using this construction one can obtain elementary 2 -simple and 2 -simplicial $d$-polytopes with arbitrarily large numbers of vertices.

\section{Examples and Results}

Now we are ready to prove our main result: that $\ell_{1}$ is in fact an extremal ray of $\mathcal{C}$. We prove this by providing three examples $W_{9}, P_{10}$, and $P_{11}$ of 2 -simple, 2 -simplicial elementary 4-polytopes with 9,10 , and 11 vertices that have simplex facets in bounded position. Using these as input for Theorem 3.7 we obtain such polytopes for arbitrarily high numbers of vertices.

4.1. Examples. For each of the following three examples we give their vertexfacet incidences together with a Schlegel diagram. Explicit rational coordinates are in Table 4.1] Calculations for the realisations were done by computer with the polymake system GJ00. Clients producing the examples below and applying the constructions, as well as coordinates for many more elementary 2-simple and 2 -simplicial 4-polytopes are available from the authors.

The combinatorial descriptions of the examples, together with many more such polytopes having a small number of vertices, were found via a complete enumeration approach using a client for the polymake system.

(1) The smallest non-trivial 2-simplicial, 2-simple 4-polytope is $W_{9}$ (see also the remark below). See Figure 4.1

(2) The second example, $P_{10}$, has 10 vertices. See Figure 4.2 Note that $P_{10}$ is not combinatorially equivalent to the 4-dimensional hypersimplex, nor its dual - although it has the same flag vector. Nevertheless, the hypersimplex itself can be obtained with the extended construction described in Remark 2.19

(3) The last example, $P_{11}$, has 11 vertices. See Figure 4.3

REMARK 4.1. $W_{9}$ and $P_{10}$ are both self-dual. $W_{9}$ is the unique non-trivial 2-simple and 2-simplicial 4-polytope with smallest number of vertices, i.e. except for the 
$\left\{v_{3}, v_{4}, v_{7}, v_{8}, v_{9}\right\}$
$\left\{v_{1}, v_{2}, v_{3}, v_{4}, v_{6}, v_{9}\right\}$
$\left\{v_{1}, v_{2}, v_{4}, v_{5}\right\}$
$\left\{v_{1}, v_{3}, v_{7}, v_{9}\right\}$
$\left\{v_{0}, v_{2}, v_{3}, v_{6}\right\}$
$\left\{v_{0}, v_{4}, v_{7}, v_{8}\right\}$
$\left\{v_{0}, v_{1}, v_{4}, v_{5}, v_{7}, v_{9}\right\}$
$\left\{v_{0}, v_{1}, v_{2}, v_{5}, v_{6}\right\}$
$\left\{v_{0}, v_{2}, v_{3}, v_{4}, v_{5}, v_{8}\right\}$
$\left\{v_{0}, v_{1}, v_{3}, v_{6}, v_{7}, v_{8}\right\}$

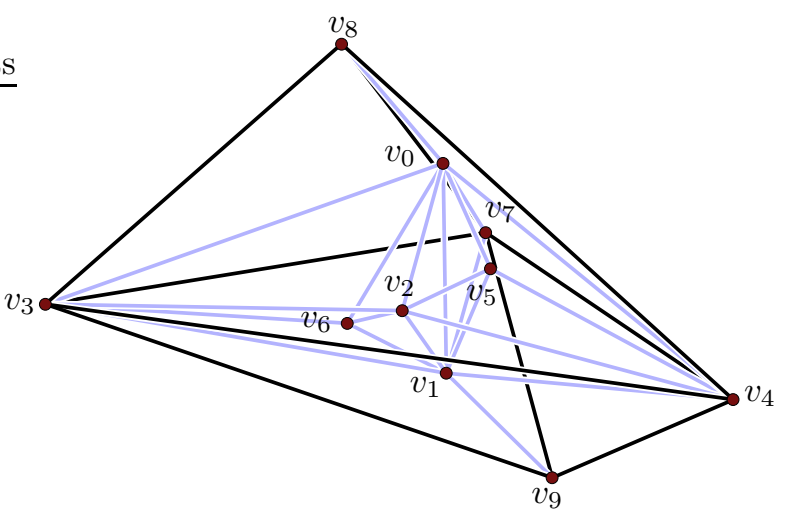

FIguRE 4.2. The vertex-facet-incidences and a Schlegel diagram of the polytope $P_{10}$.
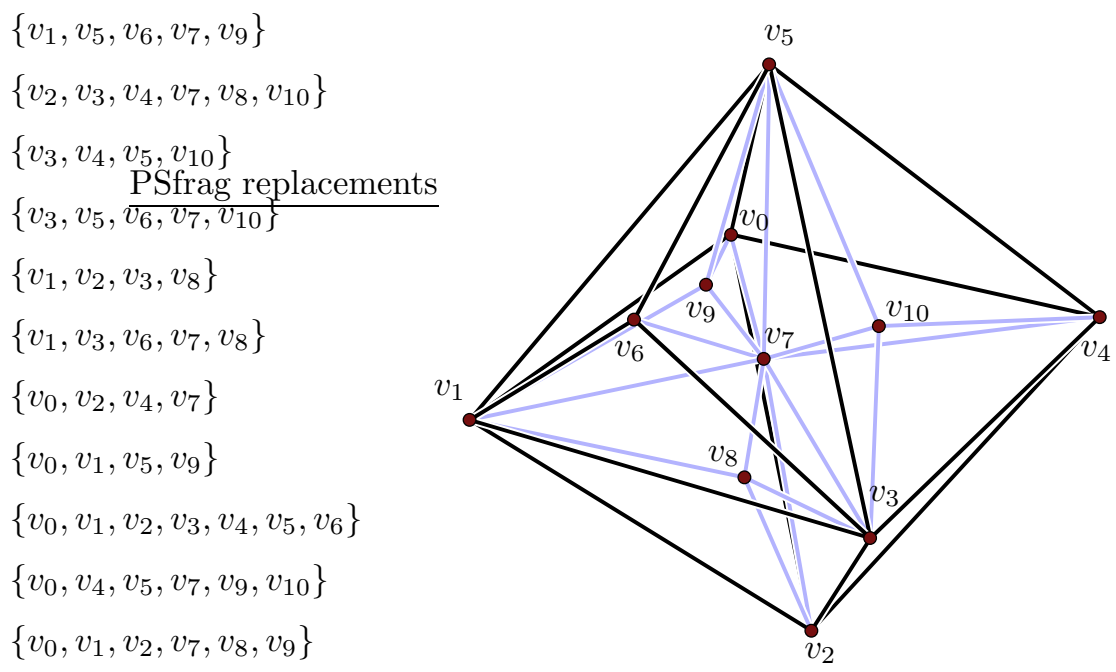

FIguRE 4.3. The vertex-facet-incidences and a Schlegel diagram of the polytope $P_{11}$.

simplex $\Delta_{4}$ there are no further 2-simplicial, 2-simple 4-polytopes with less than 10 vertices. We do not prove this here; part of the proof can be found in Paf05.

REMARK 4.2. The two examples $W_{9}$ and $P_{10}$ can in fact themselves be obtained by applying the two constructions to the 4-simplex: $W_{9}=\mathcal{I}^{2}\left(\Delta_{4} ; S\right)$ and $P_{10}=$ $\mathcal{I}^{1}\left(\Delta_{4} ; S\right)$ with an arbitrary facet $S$ of $\Delta_{4}$. Note however, that the simplex has no facet in bounded position; nevertheless, the constructions remain valid, since in 
TABLE 4.1. Coordinates for the three examples of 2 -simple and 2 -simplicial 4-polytopes that we have discussed in this section.

\begin{tabular}{|c|c|c|c|c|}
\hline$W_{9}$ & $P_{10}$ & \multicolumn{3}{|c|}{$P_{11}$} \\
\hline$(3, \quad 0, \quad 0,0)$ & $(9,-3,-3,-3)$ & 1 , & 0 & $0,0)$ \\
\hline$(1,1,1,1)$ & $(-3, \quad 9,-3,-3)$ & 0 , & 1, & $0,0)$ \\
\hline$(0,3, \quad 0,0)$ & $(-3,-3,-3,-3)$ & 0 , & 0 & $1,0)$ \\
\hline$(0, \quad 0,3,0)$ & $(-3,-3, \quad 9,-3)$ & $(-1$, & 0 & $0,0)$ \\
\hline$\left(\begin{array}{lll}0, & 0, & 0, \frac{3}{2}\end{array}\right)$ & $(-3,-3,-3, \quad 9)$ & 0 , & -1 & $0,0)$ \\
\hline$(-3, \quad 0, \quad 0,0)$ & $(1,-3,-7, \quad 1)$ & ( & 0 & $-1,0)$ \\
\hline$(0,0,-3,0)$ & $(-3, \quad 1, \quad 1,-7)$ & $\left(-\frac{11}{21}\right.$, & $\frac{11}{21}$, & $\left.-\frac{11}{21}, 0\right)$ \\
\hline$(0,-3, \quad 0,0)$ & $(3,3,3,3)$ & $\left(-\frac{11}{147}\right.$, & $\frac{11}{147}$ & $\left.-\frac{11}{147}, 1\right)$ \\
\hline$(-1,-1,-1,1)$ & $(5,-3, \quad 5, \quad 1)$ & $\left(-\frac{428}{1617}\right)$ & $\frac{428}{1617}$ & $\left.\frac{68}{147}, \frac{1}{2}\right)$ \\
\hline & $\left(\begin{array}{llll}-3, & 5, & 1, & 5\end{array}\right)$ & $\begin{array}{l}\left(\frac{68}{147},\right. \\
\left(-\frac{428}{1617}\right)\end{array}$ & $\begin{array}{l}\frac{428}{1617}, \\
-\frac{68}{147},\end{array}$ & $\begin{array}{l}\left.\frac{428}{1617}, \frac{1}{2}\right) \\
\left.\frac{428}{1617}, \frac{1}{2}\right)\end{array}$ \\
\hline
\end{tabular}

both cases the first step requires the added vertex to lie in only one adjacent facet hyperplane.

Also, $P_{11}$ can be obtained via three pseudo-stacking steps in the following way. Take a 3-dimensional octahedron $O^{3}$. Choose a facet $R$ of $O^{3}$ and let $R_{1}, R_{2}$, and $R_{3}$ be the facets adjacent to $R$. Let $B^{3}$ be the polytope obtained from $O^{3}$ by stacking the facet $R$. Let $P B^{4}$ be the pyramid over $B_{3}$ with apex $v$ and $F_{1}, F_{2}$, and $F_{3}$ be the facets of $B P^{4}$ arising as pyramids over $R_{1}, R_{2}$, and $R_{3} . B P^{4}$ is an elementary 2 -simplicial polytope, but it is not 2 -simple.

Let $\mathcal{F}_{i}$ be the sets of facets adjacent to $F_{i}$ containing the vertex $v$. Then $\left|\mathcal{F}_{i}\right|=3$. Set $P^{(0)}:=P B^{4}$ and define polytopes $P^{(i)}:=\mathbf{P S}_{\mathcal{F}_{i}}^{F_{i}}\left(P^{(i-1)}\right)$ for $i=1,2,3$. Then $P_{11}=P^{(3)}$. Note that some facets in $\mathcal{F}_{2}$ and $\mathcal{F}_{3}$ are stacked by the previous steps, but this does not influence the result.

$P_{11}$ demonstrates that one can obtain elementary 2-simple and 2-simplicial 4polytopes by pseudo-stacking polytopes without these properties in a suitable way.

4.2. Conclusions. With these examples and the constructions of Section 3, 2simplicial and 2-simple 4-polytopes with $g_{2}=0$ can be found for almost all numbers of vertices. Here is the main theorem of this paper.

TheOREM 4.3. Elementary 2-simplicial, 2-simple 4-polytopes with $k$ vertices exist for $k=5,9,10,11$ and $k \geq 13$.

Proof. We show that for $k$ as given in the claim there exist elementary 2simplicial, 2-simple 4-polytopes with $k$ vertices that have at least one simplex facet in bounded position.

For $k=9,10,11$, the above examples $W_{9}, P_{10}$, resp. $P_{11}$ have the desired properties. Let $k \geq 13$. By induction there is such a polytope $P$ with $k-5$ or $k-4$ vertices and a simplex facet $S$ in bounded position. Then the polytope $\mathcal{I}^{1}(P ; S)$, resp. $\mathcal{I}^{2}(P ; S)$ are 
elementary 2-simplicial, 2-simple 4-polytopes on $k$ vertices by Lemma 3.4 resp. 3.6 and Corollary 3.8

Corollary 4.4. The ray $\ell_{1}$ is contained in the convex hull of all flag vectors of 4-polytopes.

There are also many 2-simplicial, 2-simple 4-polytopes with $g_{2}>0$; still the existence of a 2 -simplicial, 2-simple 4 -polytope with 12 vertices is an open question.

\section{References}

[Bay87] Margaret M. Bayer, The extended f-vectors of 4-polytopes, J. Combin. Theory Ser. A 44 (1987), no. 1, 141-151. MR 88b:52009

[BB85] Margaret M. Bayer and Louis J. Billera, Generalized Dehn-Sommerville relations for polytopes, spheres and Eulerian partially ordered sets, Invent. Math. 79 (1985), no. 1, 143-157. MR 86f:52010b

[BBS02] M. M. Bayer, Aaron M. Bruening, and Joshua D. Stewart, A combinatorial study of multiplexes and ordinary polytopes, Discrete Comput. Geom. 27 (2002), no. 1, 4963, Geometric combinatorics (San Francisco, CA/Davis, CA, 2000), MR MR1871688 (2002k:52012), arXiv:math.CO/0101076

[GJ00] Ewgenij Gawrilow and Michael Joswig, Polymake A framework for analyzing convex polytopes, Polytopes - Combinatorics and Computation (G. Kalai and G. M. Ziegler, eds.), DMV Seminar, vol. 29, Birkhäuser-Verlag, Basel, 2000, pp. 43-73.

[Grü03] Branko Grünbaum, Convex Polytopes, second ed., Graduate Texts in Mathematics, vol. 221, Springer-Verlag, New York, 2003, Prepared and with a preface by Volker Kaibel, Victor Klee and Günter M. Ziegler. MR MR1976856 (2004b:52001)

[JZ00] Michael Joswig and Günter M. Ziegler, Neighborly cubical polytopes, Discrete Comput. Geom. 24 (2000), no. 2-3, 325-344, The Branko Grünbaum birthday issue, MR MR1758054 (2001f:52019), arXiv:math.CO/9812033

[Kal87] Gil Kalai, Rigidity and the lower bound theorem. I, Invent. Math. 88 (1987), no. 1, 125-151. MR MR877009 (88b:52014)

[Kal94] _ Some aspects of the combinatorial theory of convex polytopes, Polytopes: abstract, convex and computational (Scarborough, ON, 1993), NATO Adv. Sci. Inst. Ser. C Math. Phys. Sci., vol. 440, Kluwer Acad. Publ., Dordrecht, 1994, pp. 205-229. MR MR1322063 (96b:52018)

[Paf05] Andreas Paffenholz, Constructions for posets, lattices, and polytopes, Ph.D. thesis, TU Berlin, May 2005, http://edocs.tu-berlin.de/diss/2005/paffenholz_andreas.pdf

[PZ04] Andreas Paffenholz and Günter M. Ziegler, The $E_{t}$-construction for lattices, spheres and polytopes, Discrete Comput. Geom. 32 (2004), no. 4, 601-621, MR MR2096750 (2005g:06003), arXiv:math.MG/0304492

[Sta97] Richard P. Stanley, Enumerative Combinatorics. Vol. 1, Cambridge Studies in Advanced Mathematics, vol. 49, Cambridge University Press, Cambridge, 1997, With a foreword by Gian-Carlo Rota, Corrected reprint of the 1986 original. MR 98a:05001

[Ste06] Ernst Steinitz, Über die Eulerschen Polyederrelationen, Archiv für Mathematik und Physik 11 (1906), 86-88.

[Zie95] Günter M. Ziegler, Lectures on Polytopes, Graduate Texts in Mathematics, vol. 152, Springer-Verlag, New York, 1995, MR 96a:52011.

[Zie02] - Face numbers of 4-polytopes and 3-spheres, Proceedings of the International Congress of Mathematicians, Vol. III (Beijing, 2002) (Beijing), Higher Ed. Press, 2002, pp. 625-634, MR MR1957565 (2004a:52019), arXiv:math.MG/0208073

[Zie04] , Projected products of polygons, Electron. Res. Announc. Amer. Math. Soc. 10 (2004), 122-134 (electronic) MR MR2119033, arXiv:math.MG/0407042

FU Berlin, Institut für Mathematik, Arnimallee 3, 14195 Berlin, Germany

E-mail address: paffenho@math.tu-berlin.de

TU Berlin, Institut für Mathematik, MA 6-2, Strasse des 17. Juni 136, 10623 Berlin, Germany

E-mail address: awerner@math.tu-berlin.de 